\title{
IMPORTÂNCIA DA PREPARAÇÃO FÍSICA PARA O PACIENTE COM DOENÇA DE PARKINSON
}

\author{
Mary Ellen Teixeira e Julimar Luiz Pereira
}

Especialização em Preparação Física nos Esportes, DEF, UFPR, Curitiba/PR

E-mail: maryellenteixeira@gmail.com

Acesso DOI: http://dx.doi.org/10.34059/ciejop.2019v28i1-3

\begin{abstract}
RESUMO
TEIXEIRA, M.E. e PEREIRA, J.L. Importância da preparação física para o paciente com doença de Parkinson. Revista Científica JOPEF, Vol.28, n.1, pp.29-43, 2019. A doença de Parkinson (DP) é uma doença neurodegenerativa progressiva crônica descrita pela primeira em 1897 como "paralisia do tremor". Seus sintomas são variáveis, sendo o mais característico o tremor das mãos e perda de equilíbrio e força muscular. Atinge em geral, homens acima dos 60 anos, mas existem formas que podem ocorrer em indivíduos mais jovens. A progressão dos sintomas faz com que a qualidade de vida do paciente se deteriore significativamente, levando à incapacidade na realização de atividades diárias. Além do tratamento farmacológico, o exercício físico tem sido indicado como forma de atenuar e desacelerar a progressão dos sintomas. Este trabalho tem por objetivo realizar uma revisão integrativa de literatura a respeito do assunto, na qual foram analisados artigos publicados ao longo dos últimos 10 anos, buscando assim a atualização do tema. De forma geral, foi possível perceber um efeito positivo da prática de programas específicos de exercícios físicos em pacientes com DP, com melhoria da qualidade de vida. Estudos in vitro também demonstraram o efeito protetivo do exercício físico no surgimento da doença.
\end{abstract}

Palavras-chave: Doença de Parkinson; Exercício Físico; Neuroproteção; Qualidade de vida.

\begin{abstract}
TEIXEIRA, M.E. e PEREIRA, J.L. Importância da preparação física para o paciente com doença de Parkinson. Revista Científica JOPEF, Vol.28, n.1, pp.29-43, 2019. Parkinson's disease (PD) is a chronic progressive neurodegenerative disease described by the first in 1897 as "tremor paralysis." Its symptoms are variable, the most characteristic being tremor of the hands and loss of balance and muscular strength. It usually affects men over the age of 60 , but there are forms that can occur in younger individuals. The progression of symptoms causes the quality of life of the patient to deteriorate significantly, leading to the inability to perform daily activities. In addition to pharmacological treatment, physical exercise has been indicated as a way to attenuate and slow the progression of symptoms. The objective of this work is to carry out an integrative review of literature on the subject, in which articles published over the last 10 years have been analyzed, thus seeking to update the theme. In general, it was possible to perceive a positive effect of the practice of specific physical exercise programs in patients with PD, with improvement of the quality of life. In vitro studies also demonstrated the protective effect of physical exercise on the onset of the disease.
\end{abstract}

Keywords: Parkinson's disease; Physical exercise; Neuroprotection; Quality of life.

\section{INTRODUÇÃO}

Fórum Internacional de Qualidade de Vida e Saúde - Balneário Camboriú/SC, 12 de outubro de 2019 
A doença de Parkinson (DP) é uma doença neurodegenerativa progressiva crônica descrita pela primeira vez pelo Dr. James Parkinson em 1817 como uma "paralisia do tremor". Sua prevalência é estimada em 2.802 por 100.000 pessoas na América do Norte, Europa e Austrália. É acompanhado por sintomas motores e não motores devido à perda de neurônios dopaminérgicos e não dopaminérgicos do estriado (DEMAAGD; PHILIP, 2015). Os sintomas da DP incluem tremores, bradicinesia, rigidez, instabilidade postural, padrão de marcha alterado, congelamento da marcha e déficits de coordenação motora. Outros sintomas como comprometimento cognitivo, demência, insônia, depressão, ansiedade, apatia, disfunção vesical, dor e fadiga também podem ocorrer (LAUZÉ et al., 2016).

Constitui uma doença altamente variável e os sintomas podem afetar significativamente a qualidade de vida de uma pessoa, limitar as capacidades funcionais, atividades diárias e interações sociais. Como não há testes definidos para Doença de Parkinson, o diagnóstico médico pode ser feito por meio de uma anamnese abrangente e exame físico (DEMAAG; PHILIP, 2015).

O tratamento atual da DP consiste em terapias médicas (levodopa, inibidores de catecol-O-metil transferase (COMP), drogas dopaminérgicas/não dopaminérgicas), terapia não farmacológica e neurocirúrgica (estimulação cerebral profunda) (JANKOVIC; AGUILAR, 2008). Pesquisas sugerem que a atividade física também pode ser adicionada à terapia para limitar a incapacidade e melhorar a qualidade de vida. Ao longo dos últimos anos, muitas pesquisas têm sido desenvolvidas, com o objetivo de avaliar os efeitos da prática regular de exercícios sobre os sintomas da doença de Parkinson (LAUZÉ et al., 2016).

Este trabalho tem por objetivo realizar uma revisão integrativa de literatura a respeito do assunto, na qual foram analisados artigos publicados ao longo dos últimos 10 anos. A justificativa deste trabalho está na necessidade de manter a atualização a respeito dos trabalhos desenvolvidos na área, buscando auxiliar profissionais de diferentes áreas no desenvolvimento de tratamentos. Para a realização da revisão, os termos "doença de Parkinson" e "atividade física", e seus equivalentes em língua inglesa, foram inseridos em diferentes bases de dados. A partir dos artigos levantados, foram selecionados aqueles que se enquadravam nos critérios da pesquisa para análise.

\section{REVISÃO DE LITERATURA}

Fórum Internacional de Qualidade de Vida e Saúde - Balneário Camboriú/SC, 12 de outubro de 2019 
A doença de Parkinson é uma desordem neurodegenerativa progressiva que é caracterizada pela perda de dopamina causada pela degeneração de neurônios dopaminérgicos da substância nigra compacta. As características da doença de Parkinson incluem comprometimento motor (bradicinesia, rigidez, tremor, disfunção da marcha e instabilidade postural), comprometimento cognitivo (disfunção executiva do lobo frontal) e transtornos de humor. Em indivíduos saudáveis, o desempenho motor depende da interação entre o controle automático (inconsciente) e volitivo (cognitivo) do movimento. (MAZZONI; WEXLER, 2009; REDGRAVE et al., 2010).

Em geral, a doença começa a apresentar seus primeiros sintomas por volta dos 60 anos, mas pode começar mais cedo. Cerca de 5 a 10 por cento das pessoas com Parkinson têm uma doença de início precoce que começa antes dos 50 anos. As formas de Parkinson de início precoce são muitas vezes herdadas, embora nem sempre, e algumas têm sido associadas a mutações genéticas específicas. A doença afeta cerca de 50\% mais homens que mulheres; no entanto, pessoas mais jovens podem ser diagnosticadas com Parkinson também. Isto é referido como Parkinson de início precoce (ASCHERIO; SCHWARZSCHILD, 2016). Em casos muito raros, os sintomas parkinsonianos podem aparecer em pessoas antes dos 20 anos de idade. Esta condição é chamada de Parkinsonismo juvenil. É mais comumente visto no Japão, mas também foi encontrado em outros países (Itália ou Brasil). Geralmente começa com distonia (contrações musculares sustentadas que causam movimentos de torção) e bradicinesia (lentidão de movimento), e os sintomas geralmente melhoram com a medicação com levodopa. Parkinsonismo juvenil geralmente ocorre em famílias e às vezes está ligado a um gene mutante (FONG et al., 2009).

A deficiência produzida pela DP causa um impacto importante na vida do paciente e em sua família. A progressão da doença leva ao aumento da incapacidade de realizar as Atividades da Vida Diária (AVD), perda de independência e diminuição da qualidade de vida $(Q V)$, além de gerar prejuízos ocupacionais e socioeconômicos (MORRIS et al., 2009). Segundo a Organização Mundial de Saúde, a taxa de incidência global de DP é estimada em cerca de 4,5 a 19 por 100.000 habitantes, afetando ambos os sexos e raças (WHO, 2006). Considerando o aumento da expectativa de vida da população mundial e, consequentemente, do envelhecimento, é possível estimar um aumento no número 
de pessoas afetadas pela DP, tornando-se um importante problema de saúde pública global.

A literatura sugere que os efeitos da DP podem ser atenuados pelo tratamento farmacológico. No entanto, parece que os fármacos disponíveis para tratamento apenas proporcionam alívio dos sintomas, e não impedem a progressão da doença. Portanto, procurar tratamentos não farmacológicos para ajudar os pacientes é válido e necessário para trazer tratamento eficaz para eles (TARAZI et al., 2014).

Dentro desse contexto, a implementação de programas regulares de exercícios físicos parece ser uma alternativa viável para demonstrar benefícios nos sintomas motores e não motores da DP. Nesse caso, a síntese e expressão de neurotransmissores monoaminérgicos induzidos por exercício podem explicar a influência significativa do exercício sobre os distúrbios neuromotores e do humor. Por exemplo, no modelo animal, a síntese de ácido diidroxifenilacético (DOPAC) e ácido homovanálico (HVA), com metabólitos associados a níveis dopaminérgicos no cérebro, está fortemente relacionada à intensidade do exercício, bem como aos níveis extracelulares de dopamina após sete dias de treinamento com ratos (RUEGSEGGER; BOOTH, 2017).

Em humanos, a estimulação de um único padrão motor unilateral de flexão e extensão do tornozelo é responsável pela liberação dopaminérgica no estriado dorsal contralateral em pessoas com DP. Além disso, o nível de ativação da região do estriado devido à liberação de dopamina pode ser estimulado a aumentar com a aquisição de novos padrões motores em comparação com a realização do mesmo padrão repetido de movimento, sugerindo que a aprendizagem de novos movimentos em alguns pode contribuir para a manutenção ou aumento dos níveis dopaminérgicos de simples contração musculoesquelética (KAWASHIMA et al., 2012).

Argumenta-se também que o exercício contribui positivamente para déficits não motores (comportamento, humor, cognição) em relação à DP. A expressão de substâncias neurotróficas, em particular, o BDNF (Fator Neurotrófico Derivado do Cérebro), o fator de crescimento promotor da proliferação, sobrevivência e maturação de novos neurônios parecem ser induzidos a partir de uma ou mais sessões de exercício físico (SCMOLESKY et al., 2013; MONTEIRO-JUNIOR et al., 2015). Essa substância está relacionada ao aumento da arborização dendrítica e Fórum Internacional de Qualidade de Vida e Saúde - Balneário Camboriú/SC, 12 de outubro de 2019 
dos volumes nas áreas corticais, biogênese mitocondrial a partir da estimulação da expressão do fator de transcrição PGC-1a (co-ativador do receptor ativado por proliferador de peroxissoma gama alfa1) (HANDSCHIN et al., 2003), bem como, tem um papel essencial de co-regulação do sistema serotoninérgico. Portanto, esses e outros fatores neurotróficos exibem uma ampla função de plasticidade e resiliência no espectro negativo da doença, e podem ser modificados com uma dose adequada de exercício físico (DUMAN et al., 2000).

\section{RESULTADOS}

$\mathrm{Na}$ última década, acumulou-se evidência para o papel do exercício na melhoria do desempenho motor, que pode incluir a facilitação do controle cognitivo e automático do movimento. Os benefícios da prática de atividade física regular por pacientes com Parkinson, com programas especialmente desenvolvidos para suas necessidades, têm demonstrado sua eficácia na melhora dos sintomas e qualidade de vida. Nesta sessão serão analisados os trabalhos selecionados com enfoque neste tema.

Dibble et al., (2009) avaliaram as mudanças na produção de força muscular, medidas clínicas de bradicinesia e qualidade de vida após 12 semanas de um programa de exercícios de resistência excêntrica de alta intensidade em pessoas com DP leve a moderada. Vinte indivíduos com DP idiopática foram pareados em um grupo experimental ou controle ativo. Todos os participantes foram testados antes e após um período de intervenção de 12 semanas. O grupo experimental realizou contrações de quadríceps de alta intensidade em um ergômetro excêntrico 3 dias por semana durante 12 semanas. $O$ grupo controle ativo participou de um programa de exercícios baseado em evidências de DP. As variáveis de desfecho foram a força muscular do quadríceps, as medidas clínicas de bradicinesia (velocidade da marcha, tempo esgotado e ida) e a qualidade de vida específica da doença (Parkair disease questionairre-39 [PDQ-39]). Os dados foram analisados utilizando ANOVAs separadas de 2 (grupo) x 2 (período de tempo). Os resultados demonstraram um tempo significativo pelos efeitos de interação do grupo para a velocidade da marcha, timed up e go, e o escore composto PDQ-39 $(p<0,05)$. Força muscular, bradicinesia e QV foram melhorados em maior grau naqueles que realizaram treinamento de resistência excêntrica de alta intensidade em comparação com um grupo de controle ativo. Pesquisas adicionais são necessárias para Fórum Internacional de Qualidade de Vida e Saúde - Balneário Camboriú/SC, 12 de outubro de 2019 
determinar se esse tipo de treinamento tem impacto de longo prazo e se resulta em uma alteração na história natural da mobilidade e no declínio da QV em pessoas com DP.

Tanaka et al., (2009) analisaram os efeitos de um programa de exercício físico multimodal na função executiva (FE) em idosos com doença de Parkinson. A FE dos idosos foi avaliada por testes neuropsicológicos e por variáveis confusionais como atenção, sintomas depressivos e ansiedade, antes e após a intervenção. Os 20 participantes foram alocados nos Grupos Controle (GC) e Treinado (GT). O GT participou de treinamento físico generalizado por 6 meses. A ANOVA mostrou uma interação significativa $(p<0,05)$ que indicou uma contribuição benéfica do treinamento em FE. Não foram encontradas interações significativas nos resultados para variáveis de confusão entre os grupos e pré e pós-intervenção, o que apoia os resultados benéficos do treinamento físico na FE.

Taijiri et al., (2010) avaliaram os efeitos protetivos do exercício compulsivo no modelo de ratos com doença de Parkinson. Antes da lesão da 6-hidroxidopamina (6OHDA, $20 \mu \mathrm{g}$ ) no estriado direito de ratos SD do sexo feminino, a bromodeoxiuridina (BrdU) foi injetada para marcar as células em proliferação. Posteriormente, 24 horas após a lesão, os ratos foram forçados a correr na esteira ( 5 dias/semana, $30 \mathrm{~min} / \mathrm{dia}$, $11 \mathrm{~m} / \mathrm{min}$ ). Como avaliações comportamentais, o teste do cilindro foi realizado em 1 , 2, 3 e 4 semanas e o teste rotacional induzido por anfetamina foi realizado em 2 e 4 semanas com consequente eutanásia para investigações imuno-histoquímicas. 0 grupo de exercícios apresentou melhor recuperação comportamental no teste do cilindro e diminuição significativa no número de rotações induzidas por anfetaminas, em comparação com o grupo sem exercício. Correspondentemente, foi demonstrada a preservação significativa de fibras positivas para tirosina hidroxilase (TH) no estriado e neurônios positivos para HT na pars compacta da substância negra (SNc), em comparação com o grupo sem exercício. Além disso, o número de células positivas para BrdU e Doublecortin migradas para o estriado lesionado foi aumentado no grupo de exercício. Além disso, o fator neurotrófico derivado do cérebro e o fator neurotrófico derivado da linhagem glial aumentaram no estriado por meio do exercício. Os resultados sugerem que o exercício exerce efeitos neuroprotetores ou aumenta a diferenciação neuronal no modelo de doença de Parkinson em ratos, com melhora subsequente da função motora deteriorada. 
Ahlskog (2011) discute o papel neuroprotetor do exercício vigoroso na DP. Segundo o autor, evidências crescentes sugerem que o exercício vigoroso e a aptidão física em curso podem influenciar favoravelmente a progressão da DP. Modelos animais parkinsonianos revelam proteção relacionada ao exercício de neurotoxinas dopaminérgicas, aparentemente mediada por fatores neurotróficos do cérebro e neuroplasticidade (prevista a partir de estudos in vitro). Da mesma forma, o exercício melhora consistentemente a cognição em animais, também ligada à neuroplasticidade aumentada e ao aumento da expressão do fator neurotrófico. Nestes modelos animais, a imobilização tem $o$ efeito oposto. $O$ fator neurotrófico derivado do cérebro (BDNF) pode mediar pelo menos parte desse benefício do exercício. Em humanos, o exercício aumenta o BDNF sérico, e isso é conhecido por atravessar a barreira hematoencefálica. $O$ risco de DP em humanos é significativamente reduzido pelo exercício da meia-idade, documentado em grandes estudos prospectivos. Nenhum estudo abordou se o exercício influencia o risco de demência na DP, mas os pacientes exercitados com DP melhoram os escores cognitivos. Entre os idosos em geral, o exercício ou a aptidão física não foram associados apenas a melhores pontuações cognitivas, mas o exercício da meiaidade reduz significativamente o risco posterior de demência e comprometimento cognitivo leve. Por fim, numerosos estudos em idosos com e sem demência relataram aumento dos volumes de substância cinzenta cerebral associados à aptidão física ou ao exercício. Esses achados têm várias implicações para os clínicos de DP. 1) O exercício vigoroso contínuo e a aptidão física devem ser altamente encorajados. 2) Os programas de fisioterapia na DP devem incluir instrução e orientação estruturada e graduada em condicionamento físico para pacientes descondicionados com DP. 3) A levodopa e outras formas de terapia de reposição de dopamina devem ser utilizadas para alcançar a máxima capacidade e motivação para os pacientes manterem a boa forma.

Allen et al., (2011) realizaram uma revisão sistemática com meta-análise teve como objetivo determinar os efeitos do exercício e treinamento motor no desempenho de atividades relacionadas ao equilíbrio e quedas em pessoas com doença de Parkinson. Dezesseis ensaios clínicos randomizados e quase randomizados que avaliaram a eficácia do exercício e/ou treinamento motor contra nenhuma intervenção ou intervenção com placebo foram incluídos. As principais medidas de desfecho foram desempenho da atividade relacionada ao equilíbrio (15 Fórum Internacional de Qualidade de Vida e Saúde - Balneário Camboriú/SC, 12 de outubro de 2019 
tentativas) e quedas (2 tentativas). A estimativa combinada do efeito do exercício e treinamento motor indicou significativamente melhor desempenho da atividade relacionada ao equilíbrio, mas não houve evidência de um efeito sobre a proporção de quedas. O desempenho da atividade relacionada ao equilíbrio melhorou em maior grau nos testes de programas envolvendo treinamento de equilíbrio altamente desafiador, mas a diferença nos tamanhos de efeito não foi estatisticamente significativa. Exercício e treinamento motor podem melhorar o desempenho de atividades relacionadas ao equilíbrio em pessoas com doença de Parkinson. No entanto, mais pesquisas são necessárias para determinar se as quedas podem ser prevenidas nesta população.

Cruise et al., (2011) avaliaram os benefícios do exercício para o funcionamento cognitivo, humor e qualidade de vida específica (QV) para pessoas com DP. Vinte e oito indivíduos com DP foram alocados em um programa de intervenção de exercício (PIE, $n=15)$ ou grupo controle $(n=13)$. O grupo PIE realizou um programa de exercício aeróbico e anabólico progressivo duas vezes por semana durante 12 semanas. O grupo controle manteve seu estilo de vida habitual. O exercício demonstrou ter benefícios seletivos para o funcionamento cognitivo, melhorando a função executiva baseada no lobo frontal. Não foram demonstrados efeitos significativos para humor ou QV específica para doença. Estes resultados são consistentes com pesquisas anteriores demonstrando benefícios seletivos do exercício para função executiva entre adultos em idade normal e DP.

Lau et al., (2011) examinaram as consequências neurológicas, comportamentais e mecanicistas de longo prazo do exercício de resistência no parkinsonismo crônico experimental. Foi usado um modelo de camundongo crônico do tipo 1-metil-4-fenil-1,2,3,6-tetra-hidropiridina da doença de Parkinson com neurodegeneração moderada e observados os efeitos do exercício em esteira na coordenação de movimento e equilíbrio, alterações nos biomarcadores de neurônios dopaminérgicos funções mitocondriais e atividades dos fatores neurotróficos no sistema nigrostriatal. Os resultados do exercício foram comparados com os dos animais parkinsonianos controle e sedentários crônicos. Após 18 semanas de treinamento físico nos camundongos parkinsonianos crônicos, observamos uma dissuasão significativa na perda de células neuronais produtoras de dopamina e outros indicadores funcionais. A incoordenação deficiente de movimento e equilíbrio nos camundongos parkinsonianos crônicos também foi marcadamente reduzida Fórum Internacional de Qualidade de Vida e Saúde - Balneário Camboriú/SC, 12 de outubro de 2019 
após o exercício. Investigações mecanísticas revelaram que a recuperação neuronal e comportamental produzida pelo exercício nos camundongos parkinsonianos crônicos foi associada com uma função mitocondrial melhorada e um aumento nos níveis específicos da região cerebral de fatores neurotróficos derivado do cérebro e derivados da linhagem glial. Nossos achados indicam que o exercício não apenas produz proteção neuronal e mitocondrial, mas também aumentam os níveis do fator neurotrófico nigrostriatal nos camundongos parkinsonianos crônicos com moderada neurodegeneração. Portanto, modificar o estilo de vida com o aumento da atividade física seria uma abordagem neuroprotetora não farmacológica para evitar os processos neurodegenerativos, como demonstrado no parkinsonismo crônico experimental.

Tuon et al., (2012) avaliaram o efeito do treinamento físico sobre os marcadores de estresse neuroquímico e oxidativo foi avaliado no corpo estriado de ratos com doença de Parkinson (DP). Os animais foram divididos em quatro grupos. No primeiro, estavam os animais não treinados que foram submetidos a cirurgia simulada (USO), no segundo os não treinados com DP (UPD), no terceiro os treinados que foram submetidos a cirurgia simulada (TSO) e no quarto os treinados com DP (TPD) foram submetidos ao exercício na esteira. A DP foi induzida e 7 dias após a lesão, os animais foram submetidos a um teste de rotação e eutanásia por decapitação. A partir dos resultados observados, foi possível concluir que os efeitos do exercício na DP indicam a possibilidade de que o exercício, em certa medida, modula o status neuroquímico no estriado de ratos, possivelmente melhorando os parâmetros de estresse oxidativo.

Lima et al., (2013) realizaram uma revisão integrativa, na qual foram avaliados os resultados de ensaios clínicos randomizados e quase randomizados. Foram analisados trabalhos que avaliaram exercícios resistidos progressivos, definido como aqueles que envolviam contrações musculares repetitivas, fortes ou de esforço e progressão de carga à medida que as habilidades do participante mudavam. Como medidas de resultados foram utilizadas Medidas de força muscular (produção de força voluntária máxima) - contínua (força, torque, trabalho, EMG) ou ordinal (teste muscular manual) - e medidas de desempenho físico: tempo de sentar-levantar, velocidades de caminhada rápidas e confortáveis, Teste de caminhada de $6 \mathrm{~min}$, descida e subida de escada, a escala de confiança de equilíbrio específica de atividades, o teste Timed Up and Go e a bateria de desempenho físico de curta Fórum Internacional de Qualidade de Vida e Saúde - Balneário Camboriú/SC, 12 de outubro de 2019 
duração. No total, quatro ensaios randomizados foram incluídos, três dos quais relataram dados que poderiam ser agrupados em uma meta-análise. $O$ exercício resistido progressivo aumentou a força, e teve um efeito clinicamente interessante na capacidade de andar) entre pessoas com leve para moderar a doença de Parkinson. No entanto, a maioria dos resultados de desempenho físico não mostrou melhora clinicamente válida após o exercício resistido progressivo. Esta revisão sugere que o exercício resistido progressivo pode ser eficaz e útil em pessoas com doença de Parkinson leve a moderada, mas a transferência de benefício não ocorre para todas as medidas de desempenho físico. As evidências atuais sugerem que 0 treinamento de resistência progressiva deve ser implementado na reabilitação da doença de Parkinson, particularmente quando o objetivo é melhorar a capacidade de andar.

Shulman et al., (2013) compararam a eficácia de exercícios em esteira e exercícios de alongamento e resistência para melhorar a velocidade, a força e a aptidão da marcha de pacientes com doença de Parkinson. Foram selecionados 67 pacientes com DP e comprometimento de marcha, que foram divididos de forma aleatória em três grupos. (1) Um exercício em esteira em alta intensidade (30 minutos a $70 \%-80 \%$ da reserva de frequência cardíaca), (2) um exercício em esteira com intensidade menor (50 minutos em $40 \%-50 \%$ do coração) reserva de taxa), e (3) exercícios de alongamento e resistência (2 séries de 10 repetições em cada perna em 3 máquinas de resistência [leg press, extensão de perna e curvatura]). Estes exercícios foram realizados 3 vezes por semana durante 3 meses. Em todos os grupos foi observada melhora na resistência ao tempo de caminhada. O exercício em esteira de baixa intensidade resultou na maior melhora na velocidade da marcha. Ambos os exercícios de esteira com intensidade mais alta e baixa melhoraram a aptidão cardiovascular. Apenas os exercícios de alongamento e resistência melhoraram a força muscular. Portanto, o exercício pode melhorar a velocidade da marcha, força muscular e condicionamento físico para pacientes com doença de Parkinson. A combinação de exercícios em esteira e resistência pode resultar em maior benefício e requer mais investigação.

Tambosco et al., (2014) realizaram uma revisão sistemática com o objetivo de avaliar a eficácia e os limites do treinamento aeróbico e treinamento de força incluídos em programas de reabilitação física para pacientes com DP e definir modalidades práticas. Cinco revisões bibliográficas e trinta e um ensaios Fórum Internacional de Qualidade de Vida e Saúde - Balneário Camboriú/SC, 12 de outubro de 2019 
randomizados foram selecionados. O treinamento físico melhora a capacidade aeróbica, a força muscular, os parâmetros de marcha, postura e equilíbrio. Os programas de reabilitação devem começar o mais cedo possível, durar várias semanas e ser repetidos. Eles devem incluir treinamento aeróbico em bicicleta ou esteira e um programa de fortalecimento muscular. Há evidências de que o treinamento aeróbico e de força melhora as habilidades físicas de pacientes que sofrem da doença de Parkinson. Os programas de reabilitação devem ser discutidos com o paciente, levando em conta suas dificuldades e suas capacidades físicas.

Tuon et al., (2014) os efeitos de dois tipos de treinamento físico sobre o comportamento depressivo, e sobre os níveis de proBDNF, BDNF e seu receptor, TrkB, em um modelo de camundongo com DP. Camundongos C57BL/6 foram submetidos a 60 dias de exercício: corrida em esteira ou exercícios de força. PD foi induzida por administração estriatal de 6-OHDA $24 \mathrm{~h}$ após a última sessão de exercício físico. Sete dias após a injeção de 6-OHDA, o comportamento depressivo e o comportamento rotacional induzido por apomorfina foram avaliados. Os níveis de proBDNF, BDNF e TRKB foram medidos no corpo estriado e no hipocampo de camundongos por ensaio de immunoblotting. Os animais tratados com 6-OHDA mostraram um aumento significativo no tempo de imobilidade e comportamento rotacional em comparação com o grupo controle. Além disso, diminuições significativas nos níveis de proBDNF, BDNF e seu receptor, TrkB, foram observadas no grupo 6-OHDA. Ambos os tipos de exercício físico impediram comportamento depressivo e restauraram os níveis de proBDNF, BDNF e TrkB no corpo estriado e no hipocampo de camundongos administrados com 6-OHDA. Estes resultados demonstram que o treinamento físico foi eficaz para a neuroproteção no estriado e no hipocampo em um modelo experimental de DP.

Bueno et al., (2017) compararam a efetividade de três intervenções fisioterapêuticas utilizando o Rhythmic Cues (RC), Swiss Ball (SB) e Dual Task (DT), com ênfase no tratamento da marcha (passo e comprimento da passada, duração e velocidade), em indivíduos com DP. Foi realizado um ensaio clínico randomizado, dom 45 indivíduos divididos em três grupos. Diferenças estatisticamente significantes foram encontradas em todas as variáveis analisadas nos grupos $\mathrm{RC} e$ SB quando comparadas no pré e pós-intervenção. O grupo DT apresentou diferenças estatisticamente significantes em todas as demais variáveis. As três intervenções foram eficazes para os desfechos estudados, mas o grupo SB Fórum Internacional de Qualidade de Vida e Saúde - Balneário Camboriú/SC, 12 de outubro de 2019 
apresentou a maior magnitude de alteração (tamanho do efeito), enquanto o grupo $\mathrm{RC}$ apresentou maior melhora nas variáveis temporais da marcha (duração e velocidade).

Ahlskog (2018) realizou revisão de literatura no qual avaliou as evidências diretas do exercício aeróbico no cérebro de pacientes com DP. De acordo com o autor, é possível afirmar, com base nos estudos avaliados, que o exercício aeróbico influencia diretamente na progressão da doença, além de oferecer condicionamento vascular e melhora no equilíbrio do paciente.

Mükker e Myers (2018) analisaram a associação entre a aptidão física e o surgimento da doença de Parkinson e a associação com fatores de risco cardiovascular. Em seu estudo, mais de 7000 homens foram avaliados, por um período médio entre 6 e 12 anos. Foi possível avaliar que, além da aptidão física, a presença de fatores de risco, como o tabagismo, teve influência forte no surgimento da DP.

\section{CONSIDERAÇÕES FINAIS}

A partir da análise dos artigos selecionados para a elaboração deste trabalho, foi possível perceber que a realização de atividades físicas de forma regular apresenta diversos impactos positivos para pacientes com DP.

Um dos principais problemas observados nestes pacientes é a ocorrência de quedas constantes. O preparo físico se mostrou eficiente na redução deste problema, ao melhorar o equilíbrio e a força muscular destes pacientes. Desta forma, a qualidade de vida deles é também afetada, pois continuam com sua independência.

Outro ponto importante observado nos estudos realizados em animais está no papel neuroprotetor de exercício físico sobre o surgimento da DP. Os testes demonstraram uma menor incidência da doença em animais submetidos a prática regular de exercício físico, o que ressalta a importância da prática de atividades físicas para um envelhecimento saudável.

Outro ponto importante observado dentre os pacientes com DP é o desenvolvimento de depressão, em função da perda de mobilidade e sensação de incapacidade. Os estudos em animais também demonstraram o papel do exercício físico da prevenção da depressão entre estes pacientes. 
Diante destes achados, é possível afirmar que a adoção de programas de treinamento físico, devidamente elaborados por profissionais, é essencial no processo de reabilitação de pacientes com DP. É importante também ressaltar a importância da prática de exercícios físicos por pessoas saudáveis, como forma de surgir ou amenizar o surgimento da DP. Pessoas com histórico de DP na família devem dar especial atenção para sua saúde, em função do caráter genético que a doença pode apresentar.

\section{REFERÊNCIAS}

AHLSKOG, J. Eric. Aerobic exercise: evidence for a direct brain effect to slow Parkinson disease progression. In: Mayo Clinic Proceedings. Elsevier, p. 360-372. 2018

AHLSKOG, J. Eric. Does vigorous exercise have a neuroprotective effect in Parkinson disease? Neurology, v. 77, n. 3, p. 288-294, 2011.

ALLEN, Natalie E. et al. Balance and falls in Parkinson's disease: a meta-analysis of the effect of exercise and motor training. Movement disorders, v. 26, n. 9, p. 16051615, 2011.

ASCHERIO, Alberto; SCHWARZSCHILD, Michael A. The epidemiology of Parkinson's disease: risk factors and prevention. The Lancet Neurology, v. 15, n. 12, p. 1257-1272, 2016.

BUENO, Maria Eduarda Brandão et al. Comparison of three physical therapy interventions with an emphasis on the gait of individuals with Parkinson's disease. Fisioterapia em Movimento, v. 30, n. 4, p. 691-701, 2017.

CRUISE, K. E. et al. Exercise and Parkinson's: benefits for cognition and quality of life. Acta Neurologica Scandinavica, v. 123, n. 1, p. 13-19, 2011.

DEMAAGD, George; PHILIP, Ashok. Parkinson's disease and its management: part 1: disease entity, risk factors, pathophysiology, clinical presentation, and diagnosis. Pharmacy and Therapeutics, v. 40, n. 8, p. 504-510, 2015.

DUMAN, Ronald $S$. et al. Neuronal plasticity and survival in mood disorders. Biological psychiatry, v. 48, n. 8, p. 732-739, 2000.

FONG, Choong $\mathrm{Yi}$ et al. Juvenile parkinsonism associated with heterozygous frameshift ATP13A2 gene mutation. European journal of paediatric neurology, $v$. 15, n. 3, p. 271-275, 2011.

HANDSCHIN, Christoph et al. An autoregulatory loop controls peroxisome proliferator-activated receptor $y$ coactivator $1 \alpha$ expression in muscle. Proceedings of the national academy of sciences, v. 100, n. 12, p. 7111-7116, 2003. 
JANKOVIC, Joseph; AGUILAR, L. Giselle. Current approaches to the treatment of Parkinson's disease. Neuropsychiatric disease and treatment, v. 4, n. 4, p. 743, 2008.

KAWASHIMA, Shoji et al. Changes in striatal dopamine release associated with human motor-skill acquisition. PloS one, v. 7, n. 2, p. e31728, 2012.

LAU, Yuen-Sum et al. Neuroprotective effects and mechanisms of exercise in a chronic mouse model of Parkinson's disease with moderate neurodegeneration. European Journal of Neuroscience, v. 33, n. 7, p. 1264-1274, 2011.

LAUZÉ, Martine; DANEAULT, Jean-Francois; DUVAL, Christian. The effects of physical activity in Parkinson's disease: a review. Journal of Parkinson's disease, v. 6, n. 4 , p. $685-698,2016$.

LIMA, Lidiane Oliveira; SCIANNI, Aline; RODRIGUES-DE-PAULA, Fátima. Progressive resistance exercise improves strength and physical performance in people with mild to moderate Parkinson's disease: a systematic review. Journal of physiotherapy, v. 59, n. 1, p. 7-13, 2013.

MAZZONI, Pietro; WEXLER, Nancy S. Parallel explicit and implicit control of reaching. PLoS One, v. 4, n. 10, p. e7557, 2009.

MONTEIRO-JUNIOR, Renato S. et al. We need to move more: Neurobiological hypotheses of physical exercise as a treatment for Parkinson's disease. Medical hypotheses, v. 85, n. 5, p. 537-541, 2015.

MORRIS, Meg E. et al. Quantifying the profile and progression of impairments, activity, participation, and quality of life in people with Parkinson disease: protocol for a prospective cohort study. BMC geriatrics, v. 9, n. 1, p. 2, 2009.

MÜLLER, Jan; MYERS, Jonathan. Association between physical fitness, cardiovascular risk factors, and Parkinson's disease. European journal of preventive cardiology, v. 25, n. 13, p. 1409-1415, 2018.

OUCHI, Yasuomi et al. Effect of simple motor performance on regional dopamine release in the striatum in Parkinson disease patients and healthy subjects: a positron emission tomography study. Journal of Cerebral Blood Flow \& Metabolism, v. 22, n. 6, p. 746-752, 2002.

REDGRAVE, Peter et al. Goal-directed and habitual control in the basal ganglia: implications for Parkinson's disease. Nature Reviews Neuroscience, v. 11, n. 11, p. 760, 2010.

RUEGSEGGER, Gregory N.; BOOTH, Frank W. Running from disease: molecular mechanisms associating dopamine and leptin signaling in the brain with physical inactivity, obesity, and type 2 diabetes. Frontiers in Endocrinology, v. 8, p. 109, 2017.

SCHMOLESKY, Matthew T.; WEBB, David L.; HANSEN, Rodney A. The effects of aerobic exercise intensity and duration on levels of brain-derived neurotrophic factor in healthy men. Journal of sports science \& medicine, v. 12, n. 3, p. 502, 2013. 
SHULMAN, Lisa M. et al. Randomized clinical trial of 3 types of physical exercise for patients with Parkinson disease. JAMA neurology, v. 70, n. 2, p. 183-190, 2013.

TAJIRI, Naoki et al. Exercise exerts neuroprotective effects on Parkinson's disease model of rats. Brain research, v. 1310, p. 200-207, 2010.

TAMBOSCO, L. et al. Effort training in Parkinson's disease: a systematic review. Annals of physical and rehabilitation medicine, v. 57, n. 2, p. 79-104, 2014.

TANAKA, Kátia et al. Benefits of physical exercise on executive functions in older people with Parkinson's disease. Brain and cognition, v. 69, n. 2, p. 435-441, 2009.

TARAZI, F. I. et al. Emerging therapies for Parkinson's disease: from bench to bedside. Pharmacology \& therapeutics, v. 144, n. 2, p. 123-133, 2014.

TUON, T. et al. Physical training exerts neuroprotective effects in the regulation of neurochemical factors in an animal model of Parkinson's disease. Neuroscience, v. 227, p. 305-312, 2012.

TUON, T. et al. Physical training prevents depressive symptoms and a decrease in brain-derived neurotrophic factor in Parkinson's disease. Brain Research Bulletin, v. 108, p. 106-112, 2014.

WANG, Gene-Jack et al. PET studies of the effects of aerobic exercise on human striatal dopamine release. Journal of Nuclear Medicine, v. 41, n. 8, p. 1352-1356, 2000.

WORLD HEALTH ORGANIZATION. Neurological disorders: public health challenges. World Health Organization, 2006. 